\title{
The Angular Momentum Budget of the Transformed Eulerian Mean Equations
}

\author{
JOSEPH EGGER \\ Meteorologisches Institut der Universität München, Munich, Germany \\ Klaus-Peter Hoinka \\ Institut für Physik der Atmosphäre, Oberpfaffenhofen, Germany
}

(Manuscript received 26 December 2007, in final form 24 April 2008)

\begin{abstract}
The axial angular momentum (AAM) budget of zonal atmospheric annuli extending from the surface to a given height and over meridional belts is discussed within the framework of conventional and transformed Eulerian mean (TEM) theory. Conventionally, it is only fluxes of AAM through the boundaries and/or torques at the surface that are able to change the AAM of an annulus. TEM theory introduces new torques in the budget related to the vertically integrated Eliassen-Palm flux divergence and also new AAM fluxes of the residual difference circulation. Some of these torques are displayed for various annuli. In particular, the application of TEM theory generates a large positive torque at tropospheric upper boundaries in the global case. This torque is much larger than the global mountain and friction torques but is cancelled exactly by the new vertical AAM fluxes through the upper boundary. It is concluded that the TEM approach complicates the analysis of AAM budgets but does not provide additional insight. Isentropic pressure torques are believed to be similar to the TEM torques at the upper boundary of an annulus. The isentropic pressure torques are evaluated from data and found to differ in several respects from the TEM torques.
\end{abstract}

\section{Introduction}

The transformed Eulerian mean (TEM) equations attracted enormous interest and sparked intense research activities immediately after they were introduced by Andrews and McIntyre (1976). They offered new ways to look at the interaction of waves and the zonal mean flow (see Andrews et al. 1987 for a concise outline of TEM theory). One of the main technical points of this approach is the emergence of the so called Eliassen-Palm flux divergence (EPD) in the prognostic equation for zonal mean momentum. This divergence (convergence) represents the source (sink) of wave activity (see Andrews et al. 1987). Hence, it appeared to be a breakthrough that this term can be shown to be part of the zonal momentum equation. Edmon et al. (1980) expressed it succinctly, stating "the particular combinations of eddy fluxes which are represented on an Eliassen-Palm flux cross section are fundamental for the interaction be-

Corresponding author address: Joseph Egger, Meteorologisches Institut der Universität München, Theresienstr. 37, 80333 Munich, Germany.

E-mail: j.egger@lrz.uni-muenchen.de tween eddies and mean flow more so than the eddy heat and momentum fluxes considered separately."

Since then, climatologies of wave-driving have been presented (e.g., Edmon et al. 1980; Mechoso et al. 1985) and detailed correlation analyses of various terms of the TEM equations have been performed (Pfeffer 1987, 1992). Stratospheric warming events have been interpreted in terms of the TEM theory (Dunkerton et al. 1981; Palmer 1981). Randel and Stanford (1985) applied these concepts to observed baroclinic life cycles; Plumb (1986) extended the TEM approach to three dimensions. TEM theory is also discussed and applied in the oceanographic community (see, e.g., Eden et al. 2007 and references therein) and has found its way into textbooks (Pedlosky 1987; Holton 1992; Vallis 2006). There have been also critical voices. Pfeffer (1987) found that "the transient EP flux and its divergence provide much more direct information on the sources, sinks, and propagation characteristics of synoptic-scale waves in the atmosphere than they do about the response of the mean zonal current to wave action." Moreover, Pfeffer (1992) compared observed changes of the zonal mean wind with the vertical component of the Eliassen-Palm flux but did not find a correlation. 
Holton (1992) remarks in his text book that if we are "primarily concerned with the angular momentum balance for a zonal ring of air extending from the surface to the top of the atmosphere ... it proves simpler to use the conventional Eulerian mean formalism."

It is the purpose of this article to complement Pfeffer's $(1987,1992)$ approach and further explore the comments of Holton (1992) by concentrating on an aspect of this problem that has received little attention so far. Although many authors have discussed the application of TEM theory to the axial angular momentum (AAM; e.g., Edmon et al. 1980; Pfeffer 1987), the conservation form of the angular momentum equation has not been exploited. In particular, the calculations that led Holton (1992) to make his remarks on the utility of the TEM formalism in angular momentum budgets have not been published. Let us consider a zonal annulus of width $W$ and depth $D$. The AAM conservation equation in $z$ coordinates states that the AAM of this annulus can be changed only by AAM fluxes through its lateral and vertical boundaries (e.g., Egger and Hoinka 2005) and by torques at the lower boundary if the annulus intersects the topography. TEM theory reformulates the zonal momentum equation. It is of obvious interest how this transformation affects the structure of the AAM conservation equation. Which types of fluxes and torques are introduced this way? In particular, observations must be used to calculate these fluxes and torques. Are these terms large when compared to those found in standard AAM investigations?

The basic equations are given in section 2. An application to the atmospheric time mean state is presented in section 3. The discussion in section 4 includes remarks on AAM budgets in isentropic coordinates.

\section{Budget equations}

First, a brief derivation of the AAM budget equations will be given, including topography at the lower boundary. Next, the additional terms due to TEM theory will be incorporated. Readers who are hesitant to go through all these budget equations may first have a look at the simple example [(4.1) and (4.2)] presented in the discussion. A main message of this paper is contained in this example.

The angular momentum equation is

$$
\frac{\partial}{\partial t}(\rho m)+\boldsymbol{\nabla} \cdot(\rho \mathbf{v} m)=-\frac{\partial p}{\partial \lambda}+\frac{\partial \tau}{\partial z},
$$

where

$$
m=(u+\Omega a \cos \varphi) a \cos \varphi
$$

is the specific axial angular momentum. The notation is conventional, with density $\rho$, velocity $\mathbf{v}$, pressure $p$, earth's radius $a$, and $\tau$ as an angular momentum stress. It is convenient for the comparison with TEM equations to separate in (2.2) the specific relative angular momentum term

$$
m_{w}=u a \cos \varphi
$$

from

$$
m_{m}=\Omega a^{2} \cos ^{2} \varphi
$$

and to introduce for $m_{w}$ a specific prognostic equation:

$$
\frac{\partial}{\partial t}\left(\rho m_{w}\right)+\boldsymbol{\nabla} \cdot\left(\rho \mathbf{v} m_{w}\right)-f \rho v a \cos \varphi=-\frac{\partial p}{\partial \lambda}+\frac{\partial \tau}{\partial z},
$$

which follows from (2.1) after invoking the equation of continuity. Budgets for not only the AAM but also the "wind term" $\sim \rho m_{w}$ will be derived in the following because the TEM approach has been introduced to better understand the zonal mean flow predicted by (2.5).

Vertical integration of (2.1) over the depth of a layer $z_{1} \leq z \leq z_{2}$ is straightforward when both $z_{1}$ and $z_{2}$ are constant and $z_{1}$ is above the earth's topography $h$. The result is

$$
\begin{aligned}
\frac{\partial}{\partial t} \int_{z_{1}}^{z_{2}} \rho m d z+ & \nabla_{2} \cdot\left[\int_{z_{1}}^{z_{2}}\left(\rho \mathbf{v}_{2} m\right) d z\right]+\left.(\rho w m)\right|_{z_{1}} ^{z_{2}} \\
& =-\frac{\partial}{\partial \lambda}\left(\int_{z_{1}}^{z_{2}} p d z\right)+\left.\tau\right|_{z_{1}} ^{z_{2}}
\end{aligned}
$$

where $\mathbf{v}_{2}=(u, v)$ is the horizontal velocity. It is, however, also attractive for budget calculations to choose the topography as lower boundary. It follows that

$$
\begin{gathered}
\frac{\partial}{\partial t} \int_{h}^{z_{2}} \rho m d z+\nabla_{2} \cdot\left[\int_{h}^{z_{2}}\left(\rho \mathbf{v}_{2} m\right) d z\right]+(\rho w m)_{z=z_{2}} \\
=-\frac{\partial}{\partial \lambda}\left(\int_{h}^{z_{2}} p d z\right)-p_{s} \frac{\partial h}{\partial \lambda}+\left.\tau\right|_{h} ^{z_{2}},
\end{gathered}
$$

where the lower boundary condition

$$
w=\mathbf{v}_{2} \cdot \nabla h
$$

at $z=h$ has been taken into account and $p_{s}$ is the surface pressure. Zonal averaging has to face the dependence of $h$ on longitude. This rules out the application of the more elegant barycentric zonal averages (e.g., Juckes et al. 1994). Instead, we introduce the integral

$$
\tilde{b}=a \cos \varphi \int_{0}^{2 \pi} b d \lambda
$$


and the zonal average

$$
\bar{b}=\frac{1}{2 \pi} \int_{0}^{2 \pi} b d \lambda
$$

for a variable $b$. Averaged vertical integrals are written

$$
\int_{h}^{\overline{z_{2}} \rho b c d z}=\int_{\bar{h}}^{z_{2}} \bar{\rho} \bar{b} \bar{c} d z+\overline{\left(\int_{h}^{z_{2}} \rho b c d z\right)^{e}},
$$

where all deviation terms are lumped together in the "eddy" term (symbol $e$ ) and the first term on the right is called the "mean flow" term. Note that $\tilde{b}=s \bar{b}$ where $s=2 \pi a \cos \varphi$.

After zonal integration, (2.7) becomes

$$
\begin{gathered}
\frac{\partial}{\partial t} \int_{h}^{\overline{z_{2}} s \rho m d z}+2 \pi \frac{\partial}{\partial \varphi} \int_{\tilde{h}}^{z_{2}}(\cos \varphi \bar{\rho} \bar{v} \bar{m}) d z+(\bar{\rho} \tilde{w} \bar{m})_{z=z_{2}} \\
=-2 \pi \frac{\partial}{\partial \varphi} \overline{\left[\int_{h}^{z_{2}}(\cos \varphi \rho v m) d z\right]}-\overline{(s \rho w m)_{z=z_{2}}^{e}} \\
-\frac{\partial h}{p_{s} \frac{\partial \lambda}{\partial \lambda}+\overline{\left.\right|_{h} ^{z_{2}}} .}
\end{gathered}
$$

Integration of (2.12) over the width $W=a\left(\varphi_{2}-\varphi_{1}\right)$ of a zonal belt completes the derivation of the budget equation for an annulus extending from the surface to the height $z_{2}$ and from latitude $\varphi_{1}$ to $\varphi_{2}$. It is seen from (2.12) that the angular momentum of this annulus changes indeed only through mean flow and eddy fluxes at the latitudinal and upper boundaries, the mountain torque at the lower boundary, and the friction torques at both the upper and lower boundary. It is customary to omit the small upper friction torque. The surface "stress" is denoted by $-\tau_{f}$.

The flux terms in (2.12) drop out if we integrate over the globe and if $z_{2} \rightarrow \infty$. This yields the standard budget

$$
\frac{d}{d t} M=T_{o}+T_{f}
$$

where $M$ is the global axial angular momentum, $T_{o}$ is the global mountain torque, and $T_{f}$ is the global surface friction torque.

With lower boundary $z=z_{1}$ we have to replace $h$ with $z_{1}$ in the integrals in (2.12), remove the mountain torque term, and add a term $-(\bar{\rho} \tilde{w} m)_{z=z_{1}}$, on the lefthand side and a term $\overline{(s \rho w m)_{z=z_{1}}^{e}}$ on the right. The barycentric average would be suitable in this case.

The relative momentum equation, by analogy with (2.12), is

$$
\begin{gathered}
\frac{\partial}{\partial t} \int_{h}^{z_{2}} s \rho m_{w} d z+2 \pi\left[\frac{\partial}{\partial \varphi} \int_{\tilde{h}}^{z_{2}}\left(\cos \varphi \bar{\rho} \bar{v} \bar{m}_{w}\right) d z\right]+\left(\bar{\rho} \tilde{w} \bar{m}_{w}\right)_{z=z_{2}}-s f a \cos \varphi \int_{\bar{h}}^{z_{2}} \bar{\rho} \bar{v} d z-s f a \cos \varphi\left(\int_{h^{\prime}}^{z_{2}} \rho v d z\right)^{e} \\
=-2 \pi\left[\frac{\partial}{\partial \varphi} \int_{h}^{\overline{z_{2}}}\left(\cos \varphi \rho v m_{w}\right) d z\right. \\
-\overline{\left(s \rho w m_{w}\right)^{e}}-p_{s} \frac{\partial h}{\partial \lambda}-\tilde{\tau}_{f} .
\end{gathered}
$$

The Coriolis term due to the Eulerian mean meridional flow is the main new feature on the left-hand side. This term has an eddy companion. The global version of (2.14) with $z_{2} \rightarrow \infty$ also contains Coriolis torques that vanish for climatic mean conditions. Of course, (2.12) and (2.14) are closely related. For example, the mean flow Coriolis term in (2.14) is simply hidden in the second term on the left-hand side of (2.12).

It is a key point of TEM theory (Andrews and McIntyre 1976) to introduce the residual circulation

$$
\begin{aligned}
\bar{v}^{*} & =\bar{v}+\delta \bar{v}^{*} \text { and } \\
\bar{w}^{*} & =\bar{w}+\delta \bar{w}^{*},
\end{aligned}
$$

where the residual difference velocity has the components

$$
\begin{aligned}
\delta \bar{v}^{*} & =-\frac{1}{\bar{\rho}}\left[\frac{\partial}{\partial z}\left(\bar{\rho} \overline{v^{\prime} \theta^{\prime}}\right) /(\partial \bar{\theta} / \partial z)\right] \text { and } \\
\delta \bar{w}^{*} & =\frac{1}{a \cos \varphi} \frac{\partial}{\partial \varphi}\left[\cos \varphi \overline{\left.\left(v^{\prime} \theta^{\prime}\right) /(\partial \bar{\theta} / \partial z)\right] .}\right.
\end{aligned}
$$

The primes denote deviations from (2.10). As stated by Andrews and McIntyre (1976), there are many possibilities to introduce a residual circulation. We choose here the simplest version. We have to replace $(\bar{v}, \bar{w})$ in (2.12) and (2.14) by $\left(\bar{v}+\delta \bar{v}^{*}-\delta \bar{v}^{*}, \bar{w}+\delta \bar{w}^{*}-\delta \tilde{w}^{*}\right)$ and to distribute the new transport terms to both sides of (2.12) and (2.14). The result for (2.12) is 


$$
\begin{aligned}
\frac{\partial}{\partial t} \int_{h}^{\overline{z_{2}} s \rho m d z}+2 \pi\left[\frac{\partial}{\partial \varphi} \int_{\bar{h}}^{z_{2}} \cos \varphi\left(\bar{\rho} \bar{v}^{*} \bar{m}\right) d z\right]+\left(\bar{\rho} \tilde{w}^{*} \bar{m}\right)_{z=z_{2}} \\
=-2 \pi \frac{\partial}{\partial \varphi}\left[\overline{\cos \varphi\left(\int_{h}^{z_{2}} \rho v m d z\right)^{e}}-\int_{\bar{h}}^{z_{2}} \bar{\rho} \delta \bar{v}^{*} \bar{m} d z\right]-\overline{(s \rho w m)^{e}}+\left(\bar{\rho} \delta \tilde{w}^{*} \bar{m}\right)_{z=z_{2}}-\overline{p_{s} \frac{\partial h}{\partial \lambda}}-\tilde{\tau}_{f} .
\end{aligned}
$$

Integration of (2.19) over the belt width $W=a\left(\varphi_{2}-\varphi_{1}\right)$ gives the TEM formulation of the AAM budget for this annulus:

$$
\begin{aligned}
\frac{\partial}{\partial t} \int_{\varphi_{1}}^{\varphi_{2}} \int_{h}^{z_{2}} \sin d z a d \varphi+\left.2 \pi a \int_{h}^{z_{2}} \cos \varphi\left(\bar{\rho} \bar{v}^{*} \bar{m}\right) d z\right|_{\varphi_{1}} ^{\varphi_{2}}+\int_{\varphi_{1}}^{\varphi_{2}}\left(\bar{\rho} \tilde{w}^{*} \bar{m}\right)_{z=z_{2}} a d \varphi \\
=-2 \pi a\left[\left.\int_{h}^{z_{2}}(\cos \varphi \rho v m)^{e}\right|_{\varphi_{1}} ^{\varphi_{2}}-\left.\int_{\bar{h}}^{z_{2}} \cos \varphi\left(\bar{\rho} \delta \bar{v}^{*} \bar{m}\right) d z\right|_{\varphi_{1}} ^{\varphi_{2}}\right]+\int_{\varphi_{1}}^{\varphi_{2}}\left[\overline{-(s \rho w m)^{e}}+\left(\bar{\rho} \delta \tilde{w}^{*} \bar{m}\right)\right]_{z=z_{2}} a d \varphi \\
-\int_{\varphi_{1}}^{\varphi_{2}}\left(p_{s} \frac{\partial \bar{h}}{\partial \lambda}+\tilde{\tau}_{f}\right) a d \varphi .
\end{aligned}
$$

The first four terms on the right-hand side result from the vertically and meridionally integrated EPD. The first two terms stem from the integration of the meridional component of the EPD; the last two result from the vertical component. Thus, TEM theory states that the AAM budget is affected by AAM fluxes through the boundaries because of the residual circulation by Eliassen-Palm fluxes through the boundaries and by mountain and friction torques. Our terminology with respect to (2.20) calls all terms on the right-hand side torques and those on the left fluxes (except for the tendency). Following common practice, Coriolis terms are also called torques. By setting $\delta v^{*}=\delta w^{*}=0$, we recover the AAM budget of the annulus in standard form. The TEM formulation is obviously more complicated but is compatible with standard global angular momentum budgets. The global case with $z_{2} \rightarrow \infty$ leads to the correct budget Eq. (2.13).

We may switch as before from $z=h$ to $z=z_{1}$ as a lower boundary to obtain, instead of (2.20),

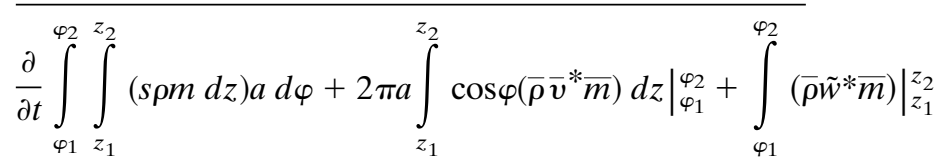

$$
\begin{aligned}
& =-\left.2 \pi a \int_{z_{1}}^{z_{2}} \cos \varphi\left[\overline{(\rho v m)^{e}}-\bar{\rho} \delta v^{*} \bar{m}\right] d z\right|_{\varphi_{1}} ^{\varphi_{2}}+\left.\int_{\varphi_{1}}^{\varphi_{2}}\left[\overline{-(s \rho w m)^{e}}+\left(\bar{\rho} \delta \tilde{w}^{*} \bar{m}\right)\right]\right|_{z_{1}} ^{z_{2}} a d \varphi .
\end{aligned}
$$

The TEM budget for the relative angular momentum is analogous to (2.20):

$$
\begin{aligned}
& \frac{\partial}{\partial t} \int_{\varphi_{1}}^{\varphi_{2}}\left(\int_{h}^{z_{2}} s \rho m_{w} d z\right) a d \varphi+\left.2 \pi a\left[\int_{\bar{h}}^{z_{2}}\left(\cos \varphi \bar{\rho} \bar{v}^{*} \bar{m}_{w}\right) d z\right]\right|_{\varphi_{1}} ^{\varphi_{2}}+\int_{\varphi_{1}}^{\varphi_{2}}\left(\bar{\rho} \tilde{w}^{*} \bar{m}_{w}\right)_{z=z_{2}} a d \varphi-\int_{\varphi_{1}}^{\varphi_{2}} s f a \cos \varphi \int_{\bar{h}}^{z_{2}} \bar{\rho} \bar{v}^{*} d z a d \varphi \\
& -\int_{\varphi_{1}}^{\varphi_{2}} s f \cos \varphi a \overline{\left(\int_{h}^{z_{2}} \rho v d z\right)^{e}} a d \varphi=-\left.2 \pi a\left[\cos \varphi \int_{h}^{\overline{z_{2}}}\left(\rho v m_{w} d z\right)^{e}-\int_{h}^{z_{2}} \cos \varphi\left(\bar{\rho} \delta \bar{v}^{*} \bar{m}_{w}\right) d z\right)\right|_{\varphi_{1}} ^{\varphi_{2}} \\
& +\int_{\varphi_{1}}^{\varphi_{2}}\left[-\overline{\left(s \rho w m_{w}\right)^{e}}+\bar{\rho} \delta \tilde{w}^{*} \bar{m}_{w}\right]_{z=z_{2}} a d \varphi+\int_{\varphi_{1}}^{\varphi_{2}} s f \cos \varphi a \bar{\rho} \overline{\left(v^{\prime} \theta^{\prime}\right)} / \partial \bar{\theta} /\left.\partial z\right|_{h} ^{z_{2}} a d \varphi-\int_{\varphi_{1}}^{\varphi_{2}}\left(\overline{p_{s} \frac{\partial h}{\partial \lambda}}+\tilde{\tau}_{f}\right) a d \varphi .
\end{aligned}
$$


As with (2.20), the first four terms on the right-hand side represent the vertically integrated form of the EPD. Note in particular that the Coriolis term on the right-hand side of (2.22) has been integrated vertically [see (2.17)] to yield torques at the upper and lower boundary. There is also a Coriolis torque due to the residual meridional wind at the left-hand side of (2.22). The global version of (2.22) is with $z_{2} \rightarrow \infty$ :

$$
\begin{aligned}
& \frac{d}{d t} M_{w}-\int_{-\pi / 2}^{\pi / 2}\left(s f a \cos \varphi \int_{\bar{h}}^{z_{2}} \bar{\rho} \bar{v}^{*} d z\right) a d \varphi= \\
& \int_{-\pi / 2}^{\pi / 2} s f a \cos \varphi\left[\int_{h}^{z_{2}}(\overline{\rho v d z})^{e}-\int_{\bar{h}}^{z_{2}} \bar{\rho} \delta \bar{v}^{*} d z\right] a d \varphi \\
& +T_{o}+T_{f} .
\end{aligned}
$$

This time, the global Coriolis torque due to the residual circulation on the left-hand side does not vanish even in the time mean, nor does the Coriolis torque due to the residual difference circulation on the right-hand side.

\section{Results}

Data have been used in the past to study (2.14) on a term by term basis at least in approximate forms (Peixoto and Oort 1992; Egger and Hoinka 2005). There is no need to repeat these calculations although the formulation of the budget Eq. (2.14) is more accurate than usual because of the proper incorporation of the lower boundary conditions. Nevertheless, this improvement is not expected to lead to a substantial revision of the results obtained so far. What has to be done, however, is to evaluate and discuss the new TEM terms on the righthand side of (2.20) and (2.22). We reduce the complexity of these terms by noting that $\bar{m} \sim \Omega a^{2} \cos ^{2} \varphi$ is an excellent approximation. With that and (2.17) and (2.18), the TEM contribution to the EPD in (2.19) is

$$
\begin{aligned}
2 \pi \frac{\partial}{\partial \varphi}\left(\cos \varphi \int_{\bar{h}}^{z_{2}} \bar{\rho} \delta \bar{v}^{*} \bar{m} d z\right)+\left(\bar{\rho} \delta \tilde{w}^{*} \bar{m}\right)_{z=z_{2}}= & 2 \pi\left[f a^{2} \cos ^{2} \varphi \bar{\rho} \overline{\left.\left(v^{\prime} \theta^{\prime}\right) /(\partial \bar{\theta} / \partial z)\right]_{z=z_{2}}}\right. \\
& +2 \pi \frac{\partial}{\partial \varphi}\left[a^{2} \Omega \cos ^{3} \varphi \bar{\rho}\left(\overline{\left.v^{\prime} \theta^{\prime}\right)} /\left(\frac{\partial \bar{\theta}}{\partial z}\right)\right]_{z=\bar{h}}\right.
\end{aligned}
$$

The vertical integration in (3.1) has been carried out using (2.17); hence, TEM theory introduces a new torque,

$$
T_{2}=\int_{\varphi_{1}}^{\varphi_{2}} 2 \pi f a^{3} \cos ^{2} \varphi\left[\bar{\rho} \overline{v^{\prime} \theta^{\prime}} /(\partial \bar{\theta} / \partial z)\right]_{z=z_{2}} d \varphi,
$$

at the upper boundary of the annulus. There is also a new torque at the lower boundary, but let us first concentrate on $T_{2}$. This torque does not vanish if we integrate over the globe so that the lower atmospheric layer of depth $\left(z_{2}-h\right)$ exchanges angular momentum with the atmosphere above. For an estimate of its order of magnitude we assume a simple profile

$$
\overline{v^{\prime} \theta^{\prime}}=A \sin 2 \varphi,
$$

where $A \sim 5-15 \mathrm{~m} \mathrm{~K} \mathrm{~s}^{-1}$ (e.g., Peixoto and Oort 1992; Juckes 2001) close to the surface and near the tropopause while $A \sim 5$ for a midtropospheric value of $z_{2}$. With $\partial \bar{\theta} / \partial z \sim 3 \times 10^{-3}\left(\mathrm{~km}^{-1}\right)$, the new torque is $T_{2} \sim$ $50 A \tilde{\rho}$ Hadley $\left(1\right.$ Hadley $\left.=10^{18} \mathrm{~J}\right)$ for the global case.
This torque is positive and implies a perpetual gain of angular momentum in the annulus. The global friction and mountain torques amount to a few Hadley (e.g., Peixoto and Oort 1992), so that the new torque dwarfs these torques. The stratosphere appears to lose a substantial amount of angular momentum according to TEM theory. Of course, the stratosphere does not contain a source of AAM and the mean fluxes of AAM through the tropopause have to vanish (Egger and Hoinka 2007). The second term on the right-hand side of (3.1) gives the torque

$$
T_{h}=\left.2 \pi a^{2} \Omega \cos ^{3} \varphi \bar{\rho}\left[\overline{\left(\boldsymbol{v}^{\prime} \theta^{\prime}\right)} / \partial \bar{\theta} / \partial z\right]_{z=h}\right|_{\varphi_{1}} ^{\varphi_{2}},
$$

which contributes little to the global budget in the longterm mean but may be quite important in midlatitude belts where $T_{h}>0(<0)$ in the Northern (Southern) Hemisphere. Moreover, the seasonal variation of $T_{h}$ is important (see Fig. 2). Note that $T_{2} \rightarrow 0$ for $z_{2} \rightarrow \infty$. Thus, TEM theory yields the smallest additional torques for deep atmospheres.

The mean torque $T_{2}$ for Northern Hemisphere win- 


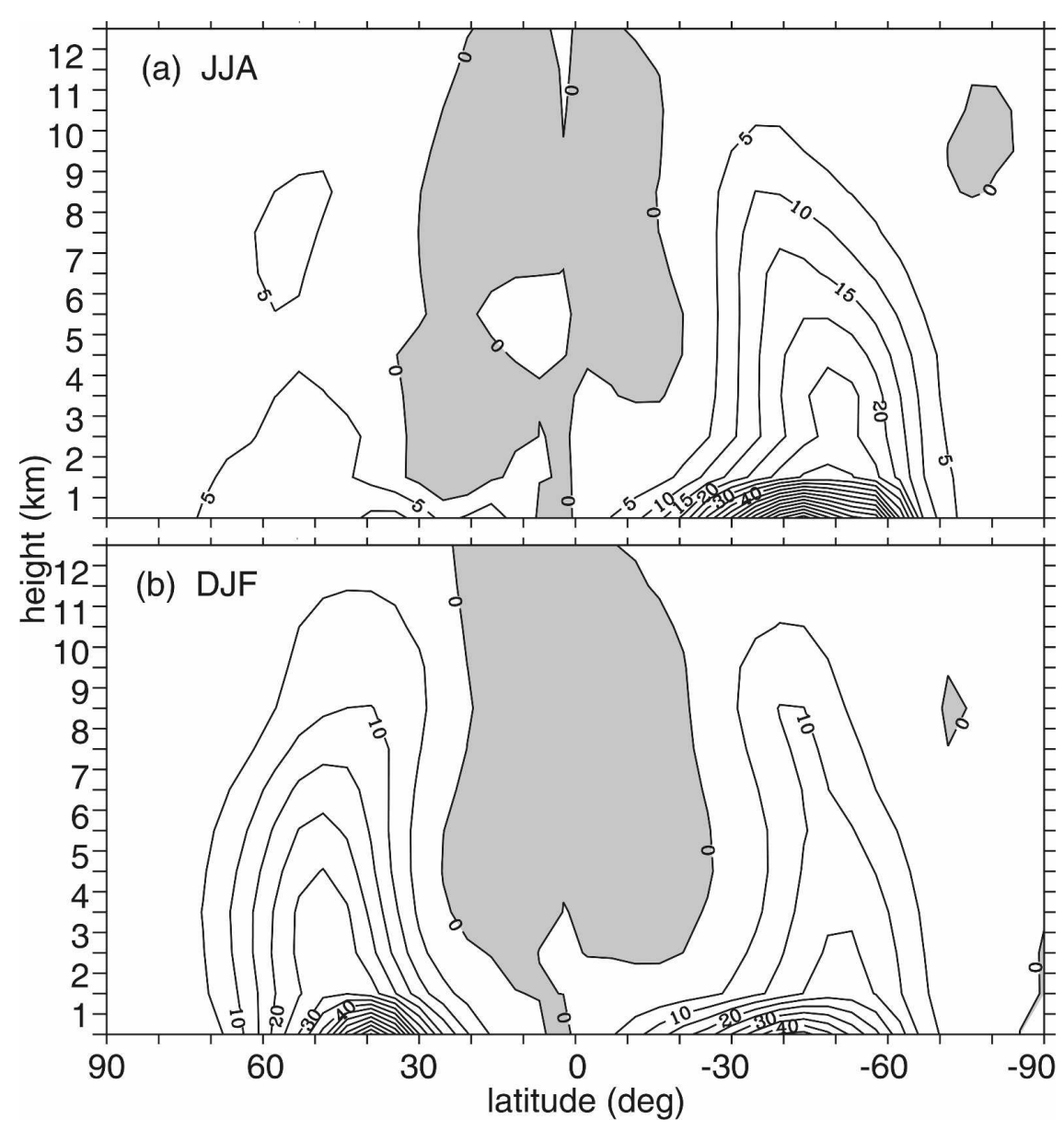

FIG. 1. TEM torque $T_{2}$ (3.2) in Hadley for Northern Hemisphere (a) summer (JJA) and (b) winter (DJF) for belts with $D \varphi=\varphi_{2}-\varphi_{1}=4.5^{\circ}$ and as a function of the height of the upper annulus boundary. All figures are based on 40-yr European Centre for Medium-Range Weather Forecasts Re-Analysis (ERA-40) data for the years 1958-2001.

ter [December-February (DJF)] and summer [JuneAugust (JJA)] is displayed in Fig. 1 for belts with $D \varphi=$ $4.5^{\circ}$ and for various annulus depths. The evaluations in Fig. 1 have been made at levels $z=z_{2}$ being $1000 \mathrm{~m}$ apart. Results have been interpolated. Torques are mostly positive and can be as large as $\sim 50$ Hadley close to the ground. Of course, the torque reflects mainly the eddy heat transport. The summer torques are almost completely restricted to the Southern Hemisphere, whereas the distribution is more symmetric in winter. The global mean of $T_{2}$ is displayed in Fig. 2 with a pronounced maximum near the ground. Global torques are $\sim 500$ Hadley close to the ground and $\sim 100$ Hadley in the midtroposphere, in reasonable agreement with (3.3). The seasonal variation of the global torques is small. Of course, similar results have been found also by others (Juckes 2001; Tanaka et al. 2004). If $z=z_{1}$ is chosen as a lower boundary, (3.1) is to be replaced by
$2 \pi \frac{\partial}{\partial \varphi}\left(\cos \varphi \int_{z}^{z_{2}} \bar{\rho} \delta v^{*} \bar{m} d z\right)+\left.\left(\bar{\rho} \delta \tilde{w}^{*} \bar{m}\right)\right|_{z=z_{1}} ^{z=z_{2}}=T_{2}-T_{1}$

where $T_{1}$ is defined by analogy to $T_{2}$. Obviously, TEM introduces the difference of large torques.

\section{Discussion}

Although it is a great attraction of TEM theory that EPD represents the eddy forcing in "terms of the potential vorticity flux, which is dynamically more fundamental than either the momentum or heat-fluxes separately" (Pedlosky 1987), we learn here that the wave forcing described by EPD is not suitable for studying the AAM budget. There is no dynamical mechanism in the atmosphere that induces the torques $T_{2}$ and $T_{h}$ or $T_{1}$. Moreover, these torques do not have any effect on 


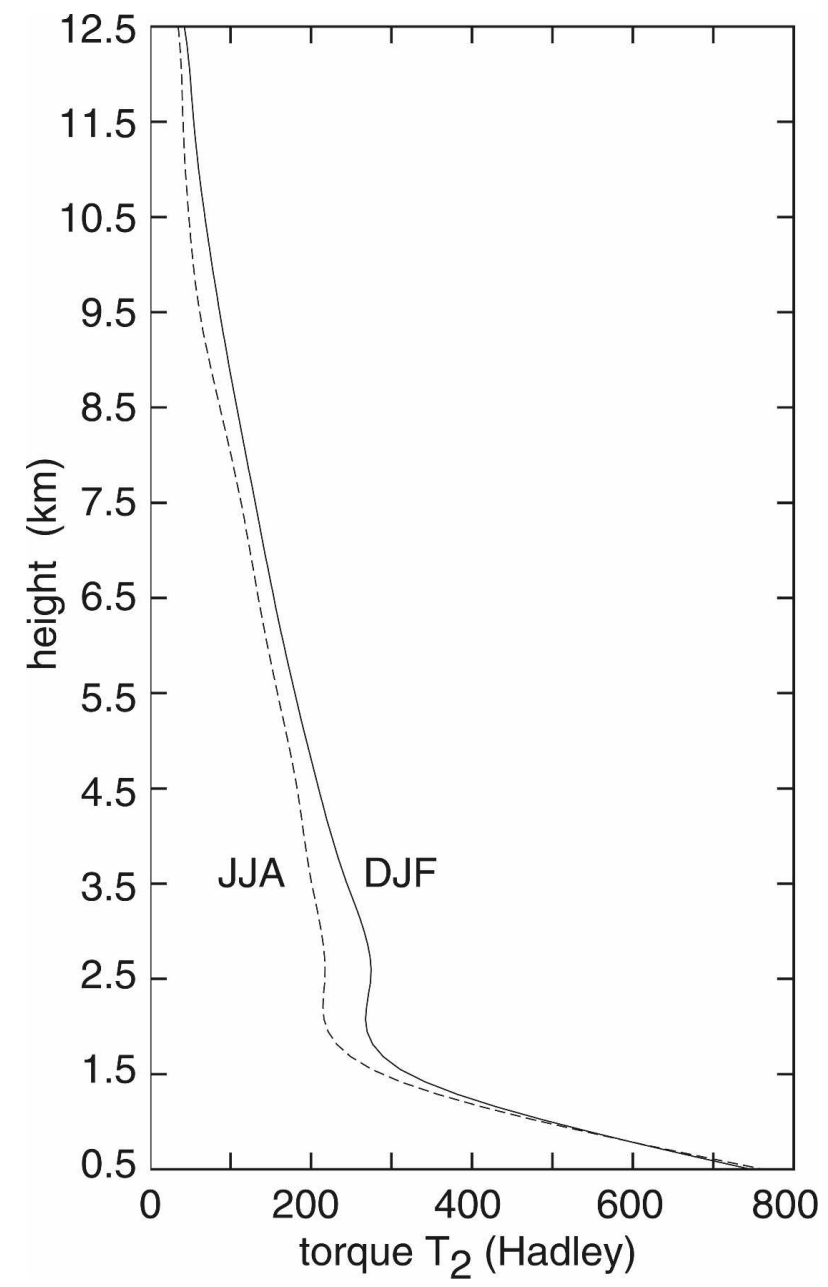

FIG. 2. Globally integrated value of $T_{2}$ in JJA and DJF in Hadley as a function of height.

the AAM of the annulus. It is hard to see what we learn from introducing such torques. They are balanced, of course, by the corresponding fluxes associated with the residual difference circulation on the left-hand sides of (2.20)-(2.22).

A similar but less general result follows from considering a two-dimensional $f$-plane model for shallow Boussinesq flow with flat lower boundary. The zonal mean flow equation is in that case

$$
\frac{\partial}{\partial t} \bar{u}-f_{o} \bar{v}+\frac{\partial}{\partial z} \overline{\left(u^{\prime} w^{\prime}\right)}=0,
$$

with $f_{o}$ constant. The switch to TEM and vertical integration yields

$$
\frac{\partial}{\partial t} \int_{z_{1}}^{z_{2}} \bar{u} d z-f_{o} \int_{z_{1}}^{z_{2}} \bar{v}^{*} d z+\left.\overline{\left(u^{\prime} w^{\prime}\right)}\right|_{z_{1}} ^{z_{2}}=f_{o} \overline{\left(v^{\prime} \theta^{\prime}\right)} /\left.(\partial \bar{\theta} / \partial z)\right|_{z_{1}} ^{z_{2}}
$$

Obviously, the terms on the right-hand side correspond with $T_{2}-T_{1}$, and we know for sure that they do not correlate with the tendency on the left-hand side. Andrews et al. (1987) point out that these terms would represent a "form drag" if the analysis were carried out on material surfaces [see also (4.9) and the related discussion]. However, (4.2) is written in $z$ coordinates where such a form drag does not exist. Note also that the results of a numerical integration of the twodimensional model with (4.1) as a zonal mean flow equation would not be affected at all by a switch to the TEM formulation. Thus, (4.2) does not provide new insights but is just more complicated than (4.1) in integrated form.

It has been pointed out by Juckes et al. (1994) that isentropic analysis offers a principal advantage over the TEM equations, namely, the "clean treatment of the lower boundary" and a "more direct portray of the diabatic heating." Moreover, the formulation of the angular momentum equations in isentropic coordinates offers a particularly clear picture of the interaction of eddies and the mean flow. In particular, Juckes et al. (1994) argued that the pressure torque [see (4.8)], which is an important part of the isentropic angular momentum balance, is closely related to $T_{2}$. They argued that

$$
\frac{1}{g} \overline{\left(p \frac{\partial M}{\partial \lambda}\right)} \approx-f \overline{\left(v_{g}^{\prime} \theta^{\prime}\right)} \cos \varphi /(\partial \bar{\theta} / \partial \bar{p})
$$

where the averages on the right have to be carried out on isobaric surfaces (see also Tanaka et al. 2004 for a somewhat different deviation of (4.3) where $v_{g}$ is the geostrophic wind). This approximation is, of course, of high interest because we have argued that $T_{2}$ cancels exactly and has no effect on the AAM budget in $z$ coordinates, whereas (4.3) suggests that $T_{2}$ is an important part of isentropic angular momentum budgets. To resolve this issue, we briefly discuss the related isentropic budget equations. The isentropic AAM equation is

$$
\frac{\partial}{\partial t} \sigma m+\nabla_{2} \cdot\left(\sigma \mathbf{v}_{2} m\right)+\frac{\partial}{\partial \theta}(\sigma \dot{\theta} m)=-\sigma \frac{\partial M}{\partial \lambda}+\frac{\partial \tau}{\partial \theta},
$$

where $\sigma=-g^{-1} \partial p / \partial \theta$ represents the density, $\dot{\theta}$ relates to the diabatic heating, and $M$ is now the Montgomery potential. Budget equations for annuli are derived by first integrating (4.4) vertically from the surface with $\dot{\theta}=\theta_{s}$ (subscript $s$ for surface values) to an isentropic surface with $\dot{\theta}=\theta_{2}$ which does not intersect the ground. The result is 


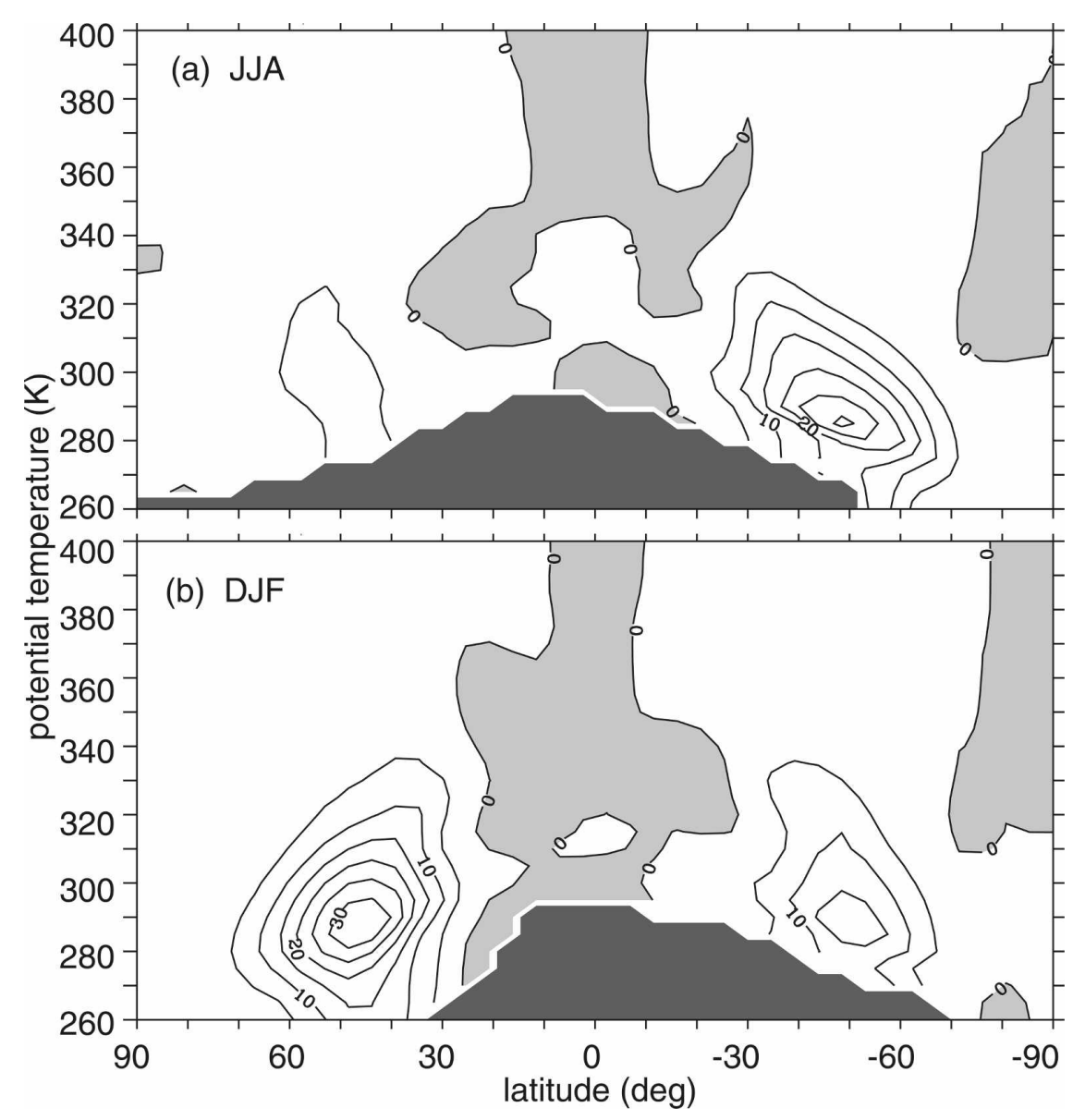

FIG. 3. Isentropic pressure torque $T_{p}$ (4.9) in Hadley for (a) JJA and (b) DJF for belts with $D \varphi=\varphi_{2}-\varphi_{1}=4.5^{\circ}$ as a function of potential temperature.

$$
\begin{aligned}
\frac{\partial}{\partial t}\left(\int_{\theta_{s}}^{\theta_{2}} \sigma m d \theta\right) & +\nabla_{2} \cdot \int_{\theta_{s}}^{\theta_{2}}\left(\sigma \mathbf{v}_{2} m\right) d \theta+\left.(\sigma \dot{\theta} m)\right|_{\theta=\theta_{2}} \\
& =-\int_{\theta_{s}}^{\theta_{2}} \sigma \frac{\partial M}{\partial \lambda} d \theta-\tau_{f}
\end{aligned}
$$

where the surface potential temperature equation

$$
\frac{\partial}{\partial t} \theta_{s}+\mathbf{v}_{2} \cdot \nabla \theta_{s}=\dot{\theta}_{s}
$$

has been invoked. There are momentum transports at the top of the layer coupled to diabatic heating. The first term on the right-hand side of (4.5) essentially represents the pressure torque. Its evaluation is easy to perform if we return to $z$ coordinates, so that

$$
\int_{\theta_{S}}^{\theta_{2}} \sigma \frac{\partial M}{\partial \lambda} d \theta=\int_{h}^{h_{2}} \frac{\partial p}{\partial \lambda} d z=\frac{\partial}{\partial \lambda} \int_{h}^{h_{2}} p d z-\left.p \frac{\partial z}{\partial \lambda}\right|_{h} ^{h_{2}},
$$

where $h_{2}$ is the geometric height of the upper isentrope. Zonal integration yields

$$
\begin{aligned}
\frac{\partial}{\partial t} \int_{\theta_{s}}^{\overline{\theta_{2}}} s \sigma m d \theta+2 \pi & \frac{\partial}{\partial \varphi}\left(\overline{\int_{\theta_{s}}^{\theta_{2}} a \cos ^{2} \varphi \sigma v_{2} m d \theta}+\widetilde{\sigma \dot{\theta} m}\right)_{\theta=\theta_{2}} \\
& =-\left.p \overline{\frac{\partial z}{\partial \lambda}}\right|_{h} ^{h_{2}}-\tilde{\tau}_{f}
\end{aligned}
$$

where the integration has to be carried out along the upper and lower surface; that is, we obtain this way a correct pressure torque term in isentropic coordinates. The mountain torque acts at the lower boundary. A direct effect of the heating is found at the upper isentrope. We arrive at (2.13) for the global case and $\theta_{2} \rightarrow \infty$.

Of course, (4.8) is not new. Johnson (1989, hereafter J89) presented, for example, a detailed observational analysis of the zonally averaged angular momentum budget at isentropes. However, a vertical integration was not carried out by Johnson (nor by Juckes et al. 


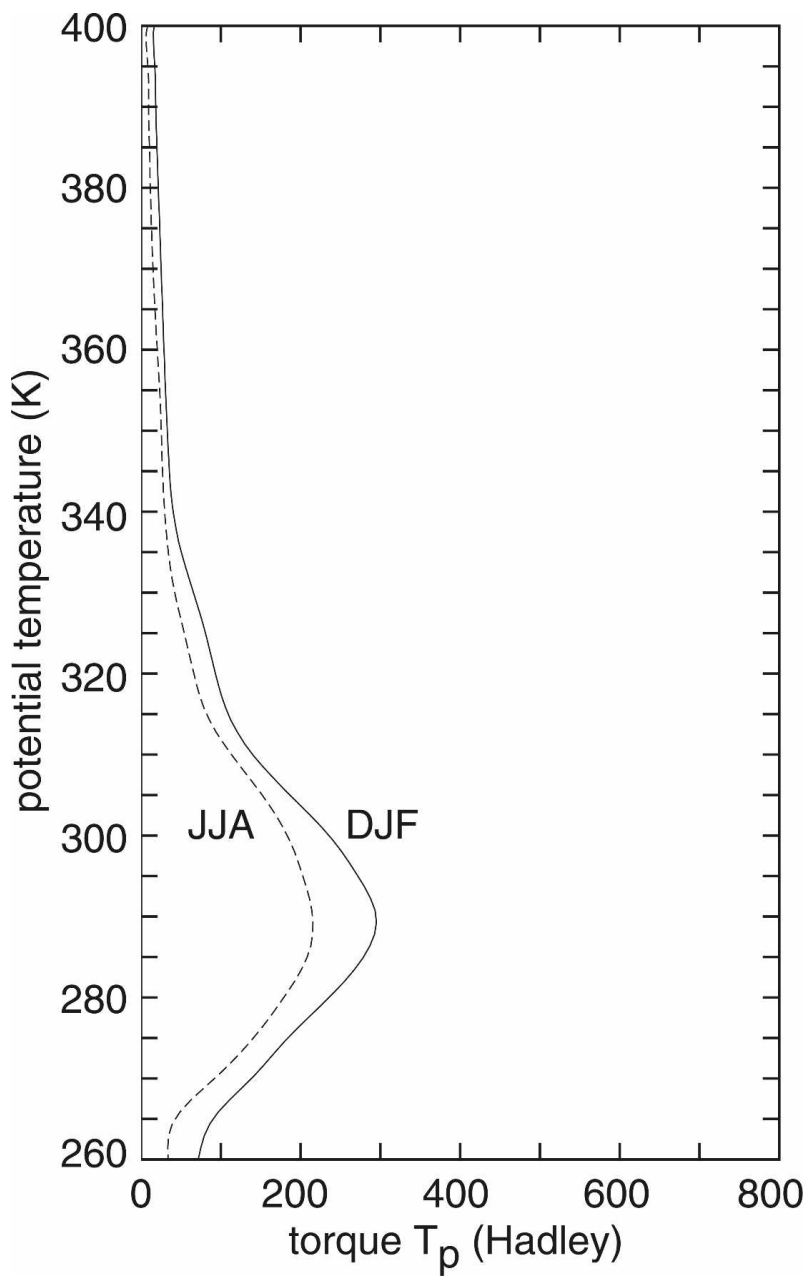

FIG. 4. Globally integrated value of $T_{p}$ in Hadley in JJA and $\mathrm{DJF}$ as a function of potential temperature.

1994), so that the role of the various terms in J89's budget differs necessarily from that in (4.8).

The pressure torque

$$
T_{p}=-\overline{\int_{\varphi_{1}}^{\varphi_{2}}\left(p \frac{\partial h_{2}}{\partial \lambda}\right)} a d \varphi
$$

is displayed in Fig. 3 for comparison with Fig. 1. The approximation (4.3), if reliable, would lead one to expect a close similarity of both figures. It is seen that these internal torques peak at midtropospheric heights at midlatitudes to decrease higher up. Maxima are $\sim 30$ Hadley. Weak negative torques are found in the tropics. This means that atmospheric eddy motion removes angular momentum from the upper troposphere at midlatitudes and brings it down to the lower troposphere. As in Fig. 1, there is a pronounced asymmetry of winter and summer cases. The patterns in Fig. 3 agree quite well with the result of J89, although we have to keep in mind that Johnson displays essentially the derivative of $T_{p}$ with respect to $\theta_{2}$, so that a zero-line in J89 is found in the midtroposphere. Moreover, the calculations of J89 are based on different data. The global mean of $T_{p}$ is presented in Fig. 4 with a maximum of $\sim 300$ Hadley in winter for $\theta=290 \mathrm{~K}$.

A comparison of Figs. 1 and 2 with Figs. 3 and 4 shows that (4.3) provides some guidance but is not fully satisfactory. In particular, $T_{2}$ has its maximum at the ground whereas $T_{p}$ peaks in the troposphere. We have to keep in mind that $T_{p}$ represents a torque that acts on the angular momentum whereas $T_{2}$ is uncorrelated with the angular momentum tendency.

Although we calculated the torque $T_{2}$ only for climatic mean conditions, it is clear that the conclusions would be the same if we applied the TEM formalism to, say, daily AAM budgets. The torque $T_{2}$ would, of course, also vary from day to day but would have no effect on the zonal mean.

Finally, it should be pointed out that the relevance of the Eliassen-Palm flux as a diagnostic tool is not restricted to its role in the zonal angular momentum budget. The relation of the flux to quasigeostrophic potential vorticity transports and wave activities is well established, and corresponding results are not at all affected by the negative outcome of our analysis, nor are any nonacceleration theorems. Nevertheless, TEM theory does not offer any advantage when it comes to analyzing AAM budgets for the atmosphere as observed.

Acknowledgments. We are grateful to the referees for constructive criticism.

\section{REFERENCES}

Andrews, D., and M. McIntyre, 1976: Planetary waves in horizontal and vertical shear: The generalized Eliassen-Palm relation and the mean zonal acceleration. J. Atmos. Sci., 33, 20312048.

— , J. Holton, and C. Leovy, 1987: Middle Atmosphere Dynamics. Academic Press, 489 pp.

Dunkerton, T., C.-P. Hsu, and M. McIntyre, 1981: Some Eulerian and Lagranigan diagnostics for a model stratospheric warming. J. Atmos. Sci., 38, 819-843.

Eden, C., R. Greatbatch, and D. Olbers, 2007: Interpreting eddy fluxes. J. Phys. Oceanogr., 37, 1282-1296.

Edmon, H., B. Hoskins, and M. McIntyre, 1980: Eliassen-Palm cross sections for the troposphere. J. Atmos. Sci., 37, 26002616.

Egger, J., and K.-P. Hoinka, 2005: Torques and the related meridional and vertical fluxes of axial angular momentum. Mon. Wea. Rev., 133, 621-633.

— 
zonal mean perspective of angular momentum. Dyn. Atmos. Oceans, 43, 100-121.

Holton, J., 1992: An Introduction to Dynamic Meteorology. Academic Press, 511 pp.

Johnson, D., 1989: The forcing and maintenance of global monsoonal circulations: An isentropic analysis. Advances in Geophysics, Vol. 31, Academic Press, 63-329.

Juckes, M., 2001: A generalization of the transformed Eulerianmean meridional circulation. Quart. J. Roy. Meteor. Soc., 127, 147-160.

_ I. James, and M. Blackburn, 1994: The influence of Antarctica on the momentum budget of the southern extratropics. Quart. J. Roy. Meteor. Soc., 120, 1017-1044.

Mechoso, C., D. Hartmann, and J. Farrara, 1985: Climatology and interannual variability of wave, mean-flow interactions in the Southern Hemisphere. J. Atmos. Sci., 42, 2189-2206.

Palmer, T., 1981: A diagnostic study of a wavenumber-2 stratospheric sudden warming in a transformed Eulerian-mean formalism. J. Atmos. Sci., 38, 844-855.

Pedlosky, J., 1987: Geophysical Fluid Dynamics. Springer, 710 pp.
Peixoto, J., and A. Oort, 1992: Physics of Climate. Springer, 520 pp.

Pfeffer, R., 1987: Comparison of conventional and transformed Eulerian diagnostic in the troposphere. Quart. J. Roy. Meteor. Soc., 113, 237-254.

- 1992: A study of eddy-induced fluctuations of the zonalmean wind using conventional and transformed Eulerian diagnostics. J. Atmos. Sci., 49, 1036-1050.

Plumb, R. A., 1986: Three-dimensional propagation of transient quasi-geostrophic eddies and its relationship with the eddy forcing of the time-mean flow. J. Atmos. Sci., 43, 1657-1678.

Randel, W., and J. Stanford, 1985: The observed life cycle of a baroclinic instability. J. Atmos. Sci., 42, 1364-1373.

Tanaka, D., T. Iwasaki, S. Uno, M. Ujiie, and K. Miyazaki, 2004: Eliassen-Palm flux diagnosis based on isentropic representation. J. Atmos. Sci., 61, 2370-2383.

Vallis, G., 2006: Atmospheric and Oceanic Fluid Dynamics: Fundamentals and Large-Scale Circulation. Cambridge University Press, $745 \mathrm{pp}$. 